\title{
CARIES DENTAL Y SU RELACION CON LISOZIMA EN SALIVA
}

\author{
Dres. MANUEL BURBANO y JULIA C. DE MORENO \\ Fuentitad de Odnatologia y Lahoratarios InFF, Quita
}

Se sabl que en la saliva hay abundante lisozima ${ }^{T}$ aungue su coneentriciór varía cumsiderablemente”.

De keverdo it la tecría cquímico-bacteriaclia de la caries y it la confirmarta tosis de Seguin, del Instiluty Pasteur: "todo mitsobio que puede producir una cantidar motable de acidez a expensas de hidratos de corbono y que mede adtaptarse a la vida imacrobia, pose las cuakdades requexidas pura decalcificar y cariar lus dientes", como la lisorima destruyc o inhibe el desarrolko do algunas bat:terias, es posible पุue tota capacidud de produeir caries

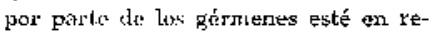
lación run ила coneutrąión baja de ljsozjma nu la saliva. Efectivamente en los trabajos de Dubois y Prevost ${ }^{3}$, parene cilce :3! vencuntró dicha relación.

Sobre estos antecedentes se organizó el siguturute plan de trabajo:

\section{MATERIALHS Y WIKTODOS}

Lus paciontos utilizadors para la preserute uvestigacion son los que concurricros a la ialla de Exadoncia de la
Facultad de Odontolomía de la Universidad Centra], y axternás niños de la Escuela "Josć Marti', de esta ciudad, en 'un total de 208 personas. En eada uno se determinó la coneertración de lissrizimia en da saliva y los resultatos se tubufarom tormando grupos de pacipnles de atuerto a la edad y sexo.

Se tamo la uuuteslra de saliva de cam da paciente, en un volumer de $3 \mathrm{cc}$, en tubos estérilen y sel unotó el númewo te piazas tariadas, obturadas $y$ ausentes, datus ton los cukles se estableció xn 'índice de caries" converscional para nuest:us fitus de experimentaciós de tipu químico-bioliggiev.

Fil "Indice de curies", st estableció, para cada paciente sumendo los valoress numéricos: de acuerdo a la siguiente uscala:

$\Lambda$ caid carie en evolución se le asignó cl valor de 1 ; Jas caries ubluradas y rote nu presemtaban indicirs de evoJución progrosiva, se las valoró con 1 leasta 3 obturaciones y con 2 cuando posiban de 4; it cacla pieza alsente, jor extracción perematura en razón de grandes caries, se asignó el valcr de 3. 
las eunecontración de lisozima en la salva se determinó con el métod, de Calleriot.

\section{RESULTADOS}

Tris la Takle I se indiun los promedios de concentración de lisn\%irna en Ia salive, labulados en grupos de edi- dé $y$ de aruerdo al ínclice de caries.

En los pacientes comprentidos ontre ti y 10 anus de odad, la cuneentratión de lisuzima asciló entre $\mathbf{3 5 , 5}$ megril ret, y $49,3 \mathrm{megm}$; ce. En extro pacientes el índice de caries fuv brjo, con uns media de 5,6 . Na se encontró una relación entrc índice de earies y *ontentración de lisozirna.

En el srupo de pacientes conipren-

TADLA I

CONCENTRACHORNS DF, LASORITRA (HIOMEDOAS) LN DDFERENTES GRUPOS DE EDADES

\begin{tabular}{|c|c|c|c|}
\hline Eidad & 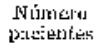 & $\begin{array}{l}\text { Indice do } \\
\text { entrifs }\end{array}$ & $\begin{array}{c}\text { Lisozima (metrict. } \\
\text { (pomedio) }\end{array}$ \\
\hline \multirow[t]{4}{*}{; -10} & 2 & 0 & 35,5 \\
\hline & 16 & $1-3$ & 49,3 \\
\hline & 28 & $4 \cdot 7$ & 35,7 \\
\hline & 7 & $x-12$ & 40,7 \\
\hline \multirow[t]{4}{*}{$11-13$} & 4 & 0 & 44,7 \\
\hline & 1.1 & $1-3$ & 42 \\
\hline & 8 & $4-7$ & 53,4 \\
\hline & 4 & $8-12$ & 54 \\
\hline \multirow[t]{5}{*}{$14-20$} & 1 & 0 & 38 \\
\hline & 5 & $1-3$ & 39,1 \\
\hline & 12 & $4-7$ & 48,2 \\
\hline & 5 & $8-12$ & 58,5 \\
\hline & 4 & $13-15$ & 23,7 \\
\hline \multirow[t]{5}{*}{$2 k \ldots$} & 26 & $1-10$ & 63,8 \\
\hline & 20 & $10 \cdot 20$ & 30,2 \\
\hline & 22 & $21-30$ & 21.0 \\
\hline & 12 & $31-40$ & 12,7 \\
\hline & 10 & $41-50$ & 13,1 \\
\hline
\end{tabular}




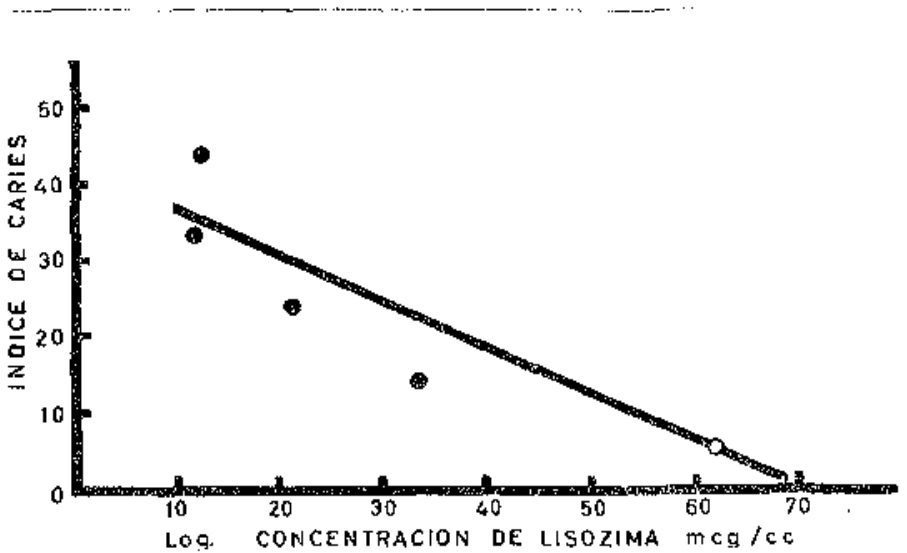

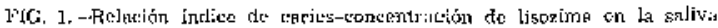
des parjentes miyores de 21 intos dil edad.

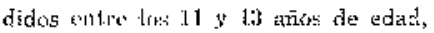

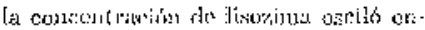

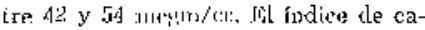

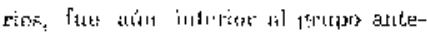

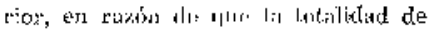

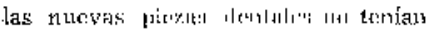

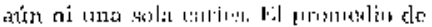

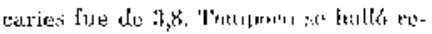

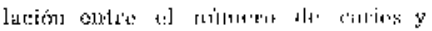

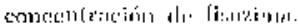

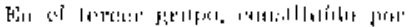

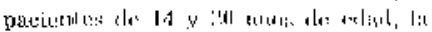

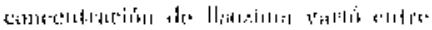

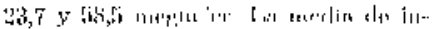

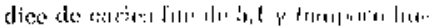
bo relactón

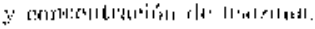

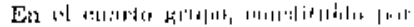

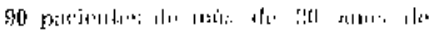

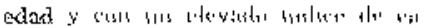

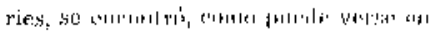

ia l'ig. 1, una tharn relación enire númern de citics y cumesutrención de lisozmma. Elecilivamenliz, a una mayor cancentanción de lisozimá corvespondíc el menor írtice de caries e inversumonte. La relución es de tipo lineal.

Cuondo lus resultades se tabularon de acuerda al sexo ke los pacientes, se rtucuatró que no hay diferencia do cuncentración de lisuzima entre lrom. bres o mujeres.

\section{DISCUSION}

Cimino se re, hasta los 20 años de codud no prlece haber una relación czlee el judice de caries y la lisozima

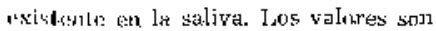
misy variables e mopendicrites de "liat y saxer. En el grupo de pacientes 
de 21 iños ton ardromte, lus resultados parecen denostrar utla eorrelación invamintente proporckonal entre con. centratión dè lisistima en là saliva y frectutiteia de caries.

\section{RESUMEN}

Biolúggicattrent.e se deterininó lit cuncentración de lizirzina an la saliva de zos pacienies, así cumu se vaIoró, em cado uno de ellos, ol "frudice de caries".

lín peritentes menores de 20 años rle edad, la coneontraciún de lisuzimia us-

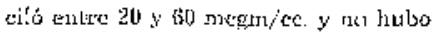
rclación con el indice do uries.

En pacientes de mayor edad, se on-

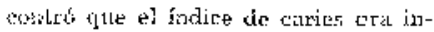
Fersumente proporcional a ta roncousirixiśn le lisnzima.

No hubo diferencias t:nlrc hombresi $y$ mujeres.

\section{SUTWMRY}

lity a bislogical method the eoncenlation of lysozyne in salival was inn vestigated in 208 patients and its rola- tion with a "dental earies index" was studied.

It was fround that in patiends under 2t] years obl of age concentration of lysoryme varied betwen 20 and $60 \mathrm{rncgm} /$ cc. and there was rot any evidence of relation with the tooth decay index.

In patients wor the 20 years of age the torkth decay index had an inverse reliationship with the unnecntration of lysorsyme.

There was no diforellecs in concerltration of lyazyme among men and wornen.

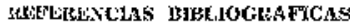

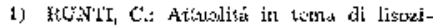
ma: esuctura allivid biolusibs of far.

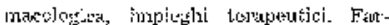

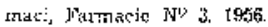

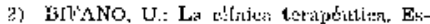
t:ariln dal Vol, 13 Tisc. 2, ז11. 259-174, ígosw, 1057

i) DUEOLS y RRFvoST: Azúteat lisozimí -

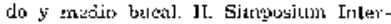

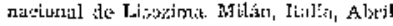
4. 1961 .

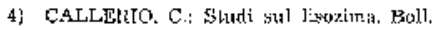
Sisc. Mod. Chir. J'avia. 44: 23T, $1932 ; y$ Antus dol Priner Sinnosizin de Yiso. xima. 